\title{
Volumetric Coronary Plaque Composition using Intravascular Ultrasound: Three-dimensional Segmentation and Spectral Analysis
}

\author{
JD Klingensmith, A Nair, BD Kuban, DG Vince \\ The Cleveland Clinic Foundation, Cleveland, USA
}

\begin{abstract}
Intravascular ultrasound provides precise tomographic assessment of coronary artery disease, allowing unique potential for analysis of both plaque geometry and composition, two critical factors related to the likelihood of plaque rupture. A novel three-dimensional segmentation technique and spectral analysis are used to create a unique tool for volumetric assessment of plaque composition.

The semi-automated $3 D$ segmentation technique was used to identify luminal and medial-adventitial borders in ECG-gated images created from radiofrequency (RF) IVUS data acquired during automated pullbacks in patients. Spectral analysis was applied to the RF data within the segmented plaque. Color-coded pseudo-histology images were created from these plaque component predictions using statistical classification trees. Quantitative analysis and visualization techniques were used to assess volumetric plaque composition and provide a unique tool for evaluation of plaque vulnerability.
\end{abstract}

\section{Introduction}

Recent evidence suggests that the likelihood of acute coronary syndromes, such as myocardial infarction, is related more to the geometric features and composition of atherosclerotic plaque than the degree of stenosis alone. Clinical studies have shown that positive vascular remodeling, a phenomenon in early atherosclerosis where an increase in the amount of plaque does not impede the lumen, but rather enlarges the vessel, is correlated with acute clinic syndromes [1]. The composition of an atherosclerotic plaque is also known to be indicative of its vulnerability [2].

Angiography, the traditional imaging modality for coronary arteries, provides a silhouette of the residual lumen and little information about the vessel wall. However, the ability to image the entire arterial wall is critical for identifying atherosclerotic plaque composition or positive remodeling. Therefore, intravascular ultrasound (IVUS), an imaging modality with the ability to provide precise tom ographic assessment of the entire coronary arterial wall, has significant potential for identification of vulnerable plaques.

We have previously developed both three-dimensional (3D) segmentation for identification of luminal and medial-adventitial (MA) borders in volumetric IVUS images [3] and spectral analysis methods for atherosclerotic plaque characterization [4]. 3D segmentation allows volumetric assessment of plaque geometry and identification of focal regions of positive vascular remodeling while spectral analysis allows identification of plaque com position. Combining spectral analysis of RF data with $3 \mathrm{D}$ segmentation provides a unique tool for volumetric assessment of plaque composition.

\section{Methods}

The semi-automated $3 \mathrm{D}$ segmentation technique was used to identify luminal and MA borders in ECG-gated images created from RF IVUS data acquired during automated pullbacks. The classification scheme developed, utilizing spectral analysis, was applied to the RF data within the plaque regions. Color-coded pseudohistology images were created based on the predicted plaque components. Quantitative analysis and visualization techniques were used to assess volumetric plaque composition.

\subsection{Data acquisition}

A custom designed ECG-gated RF data acquisition system was used for acquisition of IVUS pullback data. A $500 \mathrm{MHz}$ 8-bit analog-to-digital converter (ADC) (8500, Gage Applied Sciences, Montreal, Canada) was used to acquire the RF data. Acquisition was controlled by custom designed electronics that continuously monitored the ECG signal. Upon detection of peak $R$ wave, the system triggered the ADC to acquire enough 
scan lines for a single frame. Rotational alignment of the images was guaranteed using an 8-bit counter. Upon acquisition of the first frame, the counter was started and continuously cycled from 0 to 255. Capturing subsequent frames was triggered only when the counter returned to 0 , thus ensuring all frames would be rotationally aligned with the first frame. The scan lines were processed to determine echo information and then scan converted into IVUS images using bilinear interpolation.

The ECG-gated RF acquisition system was used in patients enrolled in trials using volumetric IVUS for assessment of the effects of lipid-lowering agents on atherosclerotic plaque burden. Automated pullbacks $(0.5$ $\mathrm{mm} / \mathrm{sec}$ ) were used to acquire volumetric IVUS data. The ECG-gated RF acquisition system was used during the automated pullback to acquire end-diastolic RF data for each. Typical pullback durations were approxim ately 90 seconds. Arterial segments lacking large sidebranches and artifacts were chosen for analysis. 82.0 \pm 29.8 enddiastolic images per pullback were segmented and analyzed for plaque composition.

\subsection{Three-dimensional segmentation}

Luminal and MA borders were identified in the sequences of RF-reconstructed pullback images using semi-automated 3D segmentation. Details of the algorithm are given in [3]. Briefly, a surface template is built automatically via a twodimensional automated edge detection algorithm [5]. The surface is warped, under the influence of forces based on the image data and the continuity of the surface model, until it converges on the borders of interest. The technique is used for both luminal and MA border detection.

The lack of a true gold standard for border location makes validation of the segmentation technique challenging. Thus, we validated the method by comparing borders detected by the segmentation technique to borders traced by a group of expert observers using Williams' index (WI), the ratio of average observer-observer variability to the average computer-observer variability. If WI is close to 1.0 , then the computer method can be considered as good as an expert human observer, because it doesn't introduce variability. If WI is too small, then the computer technique adds variability and is unacceptable.

Four expert observers traced the luminal and MA borders in 528 images acquired from 9 different patients. One useful feature of WI is that different metrics can be used for the assessment of variability, and since it is possible to have two different shapes with the same cross-sectional area, Hausdorff distance, a more strict measure of border mismatch, was used. For each point on one contour, the minimum distance to any point on another contour is noted.
The Hausdorff distance for these two contours is the maximum of all the minimum distances. Therefore, if two borders are well-aligned, the Hausdorff distance will be small.

\subsection{Spectral analysis and visualization}

Previous studies by our group have investigated the use of spectral analysis and statistical classification trees for identifying fibrous, fibro-lipidic, calcified, and calcified-necrotic regions of atherosclerotic plaque [4]. Briefly, RF data was acquired from explanted coronary arteries and corresponding histological sections were collected. Homogeneous regions-of-interest (ROIs) were identified in the histology images and a novel IVUShistology matching technique was used to identify the specific samples of the acquired scan lines corresponding to those ROIs. Spectra were calculated using various classical Fourier (FFT) and autoregressive (AR) models. A database of spectral parameters was builtand statistical classification trees were used to classify the regions of plaque. The AR models provided the best results, accurately detecting each of the 4 plaque types at rates from $84 \%$ to $95 \%$.

In the current study, after the atherosclerotic plaque was identified in the ECG-gated RF image sequences using the 3D segmentation technique, the plaque characterization algorithm was used to classify plaque components. Each sample in the plaque was classified as illustrated in Figure 1. A region-of-interest (ROI), containing portions of 7 scan lines, 64 samples each $(384$ $\mu \mathrm{m})$, was defined for each sample in the plaque. The spectra of each scan line portion was calculated using a $10^{\text {th }}$ order autoregressive model. These spectra were

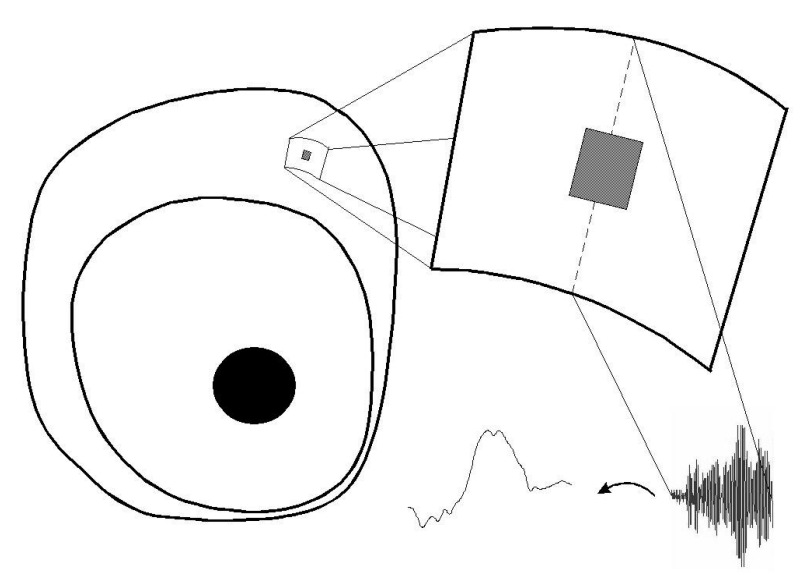

Figure 1. Illustration of plaque characterization scheme used to create pseudo-histology images. 


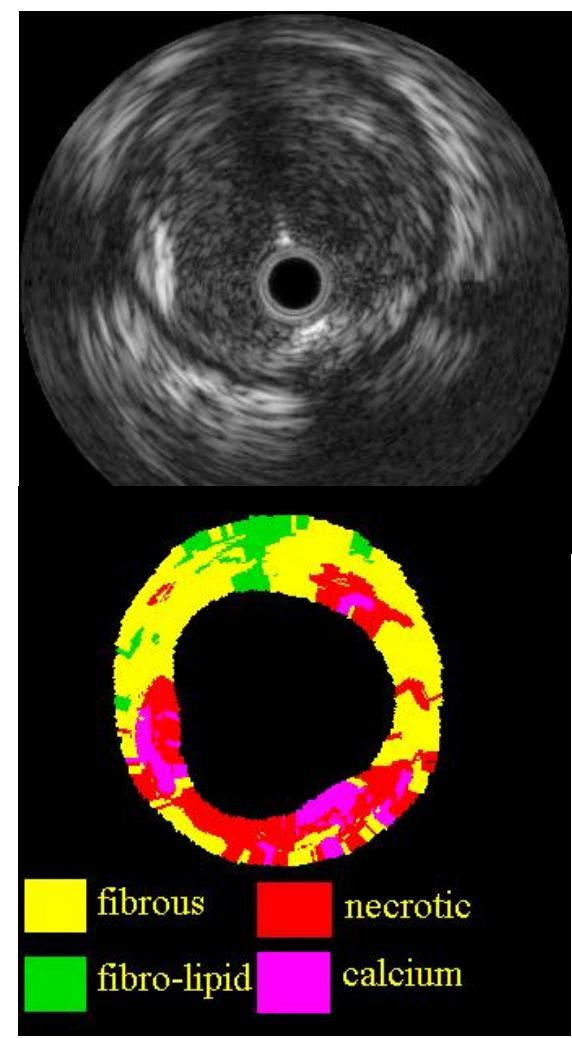

Figure 2. Example RF image and corresponding pseudo-histology color coded image.

averaged together and normalized by a spectrum calculated using a perfect reflector. Seven param eters of the normalized spectrum were fed into the statistical classification tree that was built from the database of spectral signatures. "Pseudo-histology" images, representing the plaque composition, were created. An example is shown in Figure 2.

A volum etric data set of histological composition was created by applying the RF plaque classification to each end-diastolic image in the sequences resulting from the ECG-gated pullbacks. The luminal surfaces created from the detected luminal borders were then color-coded according to the histological components, allowing a unique fly-through visualization of the inside of the coronary lumen, where the color of the inside of the arterial wall denotes the most superficial histological feature.

The volumetric data set also provided a unique quantitative assessment of both arterial geometry and composition. Plots of lumen, vessel, and plaque crosssectional area versus image slice were created for geometric assessment and identification of positive remodeling. The plaque area $w$ as then sub-divided in to histological components and plotted versus image slice,

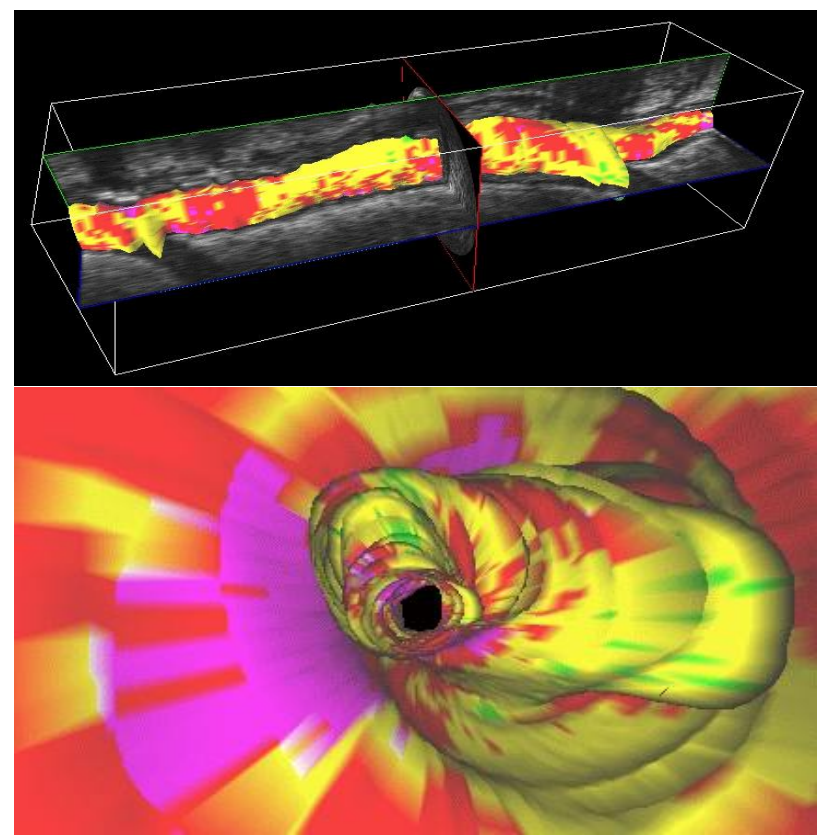

Figure 3. Example detected luminal surface with histology color coding (above) and fly-through view (below).

creating a volumetric assessment of plaque composition previously unavailable.

\section{Results}

W I (for Hausdorff distance) and the $95 \%$ confidence interval for luminal border detection in the 528 clinical images was $0.98(0.89,1.06)$. For MA border detection, the WI was $0.99(0.95,1.04)$.

Figure 3 shows an example luminal surface, color coded with the most superficial corresponding plaque component. Also shown is an example view from the lumen fly-through. The $3 \mathrm{D}$ segmentation and spectral analysis, along with the quantitative and visualization tools, have been integrated into one software package.

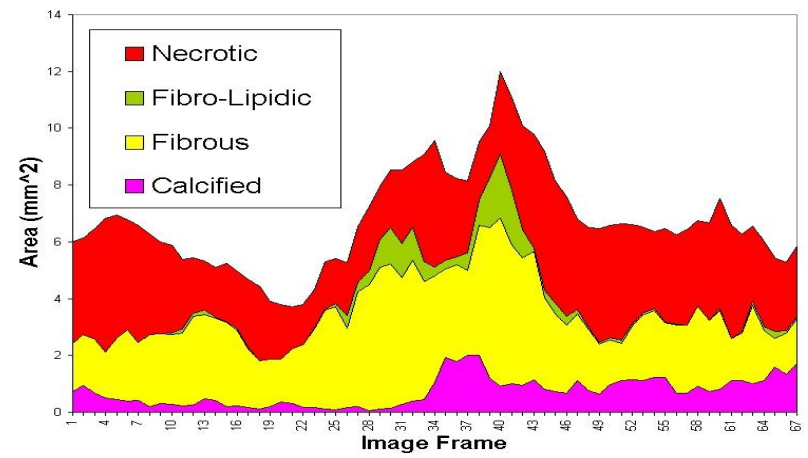

Figure 4. Plot of plaque components versus image frame. 
The user can interactively interrogate the coronary artery, inspecting the plaque components, or output quantitative measurements. Figure 4 shows an example plot of plaque area versus image frame. The relative amount of each plaque component along the length of the arterial segment is represented by the area of the corresponding colored regions in the plot.

\section{Discussion and conclusions}

W I allowed evaluation of the $3 \mathrm{D}$ segmentation technique within the context of inter-observer variability. For both luminal and MA borders, WI was nearly 1.0 (1.0 was contained in the $95 \%$ confidence intervals); therefore, the computer technique is as good at identifying the borders as an expert human observer. The validation images were identified by observing $30 \mathrm{~mm}$ arterial segments from 9 patients. Although 529 images were used for validation, a more thorough clinical validation in a larger cohort is underway.

The active surface approach to $3 \mathrm{D}$ segmentation of sequences of IVUS images is robust and produces results more reliable and reproducible than manual tracing. The semi-automated approach is also less time-consuming and tedious than manual techniques, allowing fast and intuitive identification of the luminal and MA borders in the ECGgated RF images.

By fusing 3D segmentation of ECG-gated IVUS image sequences and spectral analysis of RF data, we have provided detailed volumetric assessment of the histological components of coronary plaques. The analysis of $3 \mathrm{D}$ plaque composition and geometry has unique potential for identification of vulnerable plaques, because it can identify two of the main factors involved in the likelihood of plaque rupture, positive remodeling and plaque composition. The individual impact of these two factors is documented, but speculation remains regarding the relationship between them. The tools developed could help elucidate this relationship in vivo, determining the histological components of positively remodeled plaques, potentially providing insights into plaque rupture that were previously unavailable.

There remain limitations in this technology that have yet to be overcome. The spectral analysis algorithms were developed ex vivo using careful correspondence of IVUS and histology, which is the gold standard. However, in vivo validation of the spectral analysis is not complete. Another limitation of the system, relates to the ECG-gated acquisition. To accurately calculate plaque, or histological component volumes, the longitudinal spacing between the acquired IVUS images is required. We have developed a $2^{\text {nd }}$ generation acquisition system capable of recording the inter-frame distance and allowing accurate plaque volume calculations. In addition, the current system does not account for curvature of the artery, but ongoing work with biplane angiography and other technologies will allow for geometrically correct $3 \mathrm{D}$ reconstruction with plaque composition. Other future work possibly includes more sophisticated visualization tools. The current fly-through tool has the inside of the lumen surface colored to represent the most superficial plaque component. Traditional volume rendering and schemes for representing deeper plaque components will be investigated for visualization of the entire $3 \mathrm{D}$ plaque.

\section{References}

[1] Schoenhagen P, Ziada KM, Kapadia SR, Crowe TD, Nissen SE, Tuzcu EM. Extent and direction of arterial remodeling in stable versus unstable coronary syndromes: an intravascular ultrasound study. Circulation 2000; 101(6):598-603.

[2] Falk E, Shah PK, Fuster V. Coronary plaque disruption. Circulation 1995; 92(3):657-71.

[3] Klingensmith JD, Shekhar R, Vince DG. Evaluation of three-dimensional segmentation algorithms for the identification of luminal and medial-adventitial borders in intravascular ultrasound images. IEEE Trans Med Imaging 2000; 19(10):996-1011.

[4] Nair A, Kuban BD, Obuchowski NA, Vince DG. Assessing spectral algorithms to predict atherosclerotic plaque composition with normalized and raw intravascular ultrasound data. Ultrasound Med Biol 2001; 27(10):131931.

[5] Meier DS, Cothren RM, Vince DG, Cornhill JF. Automated morphometry of coronary arteries with digital image analysis of intravascular ultrasound. Am Heart J 1997; 133(6):681-90.

Address for correspondence.

D. Geoffrey Vince, $\mathrm{PhD}$

Department of Biomedical Engineering / ND20

The Cleveland Clinic Foundation

Cleveland, Ohio 44195

vince@bme.ri.ccf.org 